\title{
Quatre nouveaux Melanoconion (Diptera, Culicidae) de la Guyane Française
}

\author{
par Jean CLASTRIER \\ Laboratoire d'Ecologie médicale et de Pathologie parasitaire et Institut National de la Santé \\ et de la Recherche médicale \\ Faculté de Médecine, F 34 -Montpellier ( $\mathrm{P}^{\mathrm{r}}$ J.-A. Rıoux)
}

\section{Résumé}

Quatre nouveaux Culex, s.g. Melanoconion, sont décrits de la Guyane Française ( $\hat{\sigma}$ seulement) : $C$. seneveti, qui prend place dans le groupe de C. phlogistus Dyar, C. inhibitator Dyar et Knab, C. wepsteri Komp et Rozeboom; C. abonnenci très proche de C. elevator Dyar et Knab; C. caudatus, qui ressemble à $C$. serratimarge Root et $C$. isabelae Duret; $C$. trigeminatus appartenant au groupe de C. dunni Dyar, C. zeteki Dyar, C. caribeanus Galindo et Blanton.

\section{Summary}

Four new species of Culex, subg. Melanoconion are described from French Guiana ( $\sigma^{*}$ only): C. seneveti, which falls into the group of $C$. phlogistus Dyar, C. inhibitator Dyar and Knab, C. wepsteri Komp and Rozeboom; C. abonnenci closely related to C. elevator Dyar and $\mathrm{Knab} ; C$. caudatus which resembles $C$. serratimarge Root and $C$. isabelae Duret ; $C$. trigeminatus belonging to the group of $C$. dunni Dyar, C. zeteki Dyar, C. caribeanus Galindo and Blanton. 
Au cours de chasses nocturnes effectuées en Guyane Française à l'aide de pièges lumineux, nous avons capturé quelques mâles du genre Culex Linné, 1758, sous-genre Melanoconion Theobald, 1903, revenant à quatre espèces nouvelles que nous décrivons ci-dessous.

C'est avec un très grand plaisir que nous dédions les deux premières, respectivement, à M. le Professeur G. Senevet, notre Maître et ancien Collègue à l'Institut Pasteur d'Algérie, ainsi qu'à M. E. Abonnenc, ancien Entomologiste à l'Institut Pasteur de Guyane.

Types provisoirement conservés dans la collection personnelle de l'auteur.

\section{Culex (Melanoconion) seneveti n. sp.}

MÂLE.

Antenne gris noirâtre; de la même longueur que la trompe. Palpe entièrement noir ; beaucoup plus long que la trompe, l'extrémité du troisième article n'atteignant pas tout à fait la base des labelles. Clypeus et trompe noirs. Occiput : en bordure des yeux, s'étendant sur le vertex, une bande d'écailles larges, plates, couchées, hyalines, à reflets variables, blanchâtres, bleuâtres ou dorés suivant l'incidence de la lumière ; postérieurement à celle-ci, une autre bande, concentrique et moins large, d'écailles également couchées, blanches, étroites, légèrement recourbées ; quelques écailles dressées, fourchues, noires, dispersées parmi les précédentes et d'autres, alignées sur un rang, à la partie la plus postérieure de l'occiput.

Mesonotum, scutellum et post-scutellum sensiblement d'un même brun uniforme, les deux premiers portant des poils et des écailles noirs. Pleures du même brun que le mesonotum dans leur partie supérieure, jusqu'au niveau d'une ligne oblique suivant le bord supérieur de la sternopleure; progressivement plus claires au-dessous de cette ligne, de telle sorte que la sternopleure, le méron, les hanches et les trochanters paraissent à peine teintés de brun. Le pronotum antérieur est au contraire nettement plus sombre que le reste du thorax. Quelques écailles hyalines au niveau des soies sternopleurales inférieures et des mésépimérales supérieures.

Aile: toutes les écailles d'un même brun léger, sauf sur la costa où elles sont noirâtres. Balancier: base et moitié basale de la tige blanchâtres, le reste de la tige et bouton noirs. Pattes : hanches et trochanters brun clair (blanchâtres sur les spécimens peu colorés), le reste entièrement brun-noir, à reflets bronzés, sauf la partie ventrale des fémurs qui est claire.

Abdomen : tergites entièrement noirs, à reflets bronzés ; sternites difficiles à voir sur les échantillons examinés, présentant des touffes basolatérales d'écailles blanches, petites et peu nettes sur les segments II et III, beaucoup plus grosses et très apparentes à partir de IV.

Pince génitale (fig. 1). Coxite nettement grossi dans ses trois quarts basaux, sur certains spécimens subglobuleux, avec les faces externe et interne fortement convexes; encore très large sur le dernier quart, au niveau du lobe subapical. Une touffe de poils longs et souples sur la face interne, au-dessus du lobe subapical. 
Les deux branches de la division interne de ce lobe courtes, particulièrement la plus interne, qui porte un filament rectiligne, terminé par un crochet long et vigoureux. Le filament de l'autre branche est doublement et irrégulièrement recourbé, anguleux, et se termine par un épaississement en crête transversale. A la base et dans la partie médiane de cette division, un groupe de poils courts et raides (sur la face cachée de la figure).

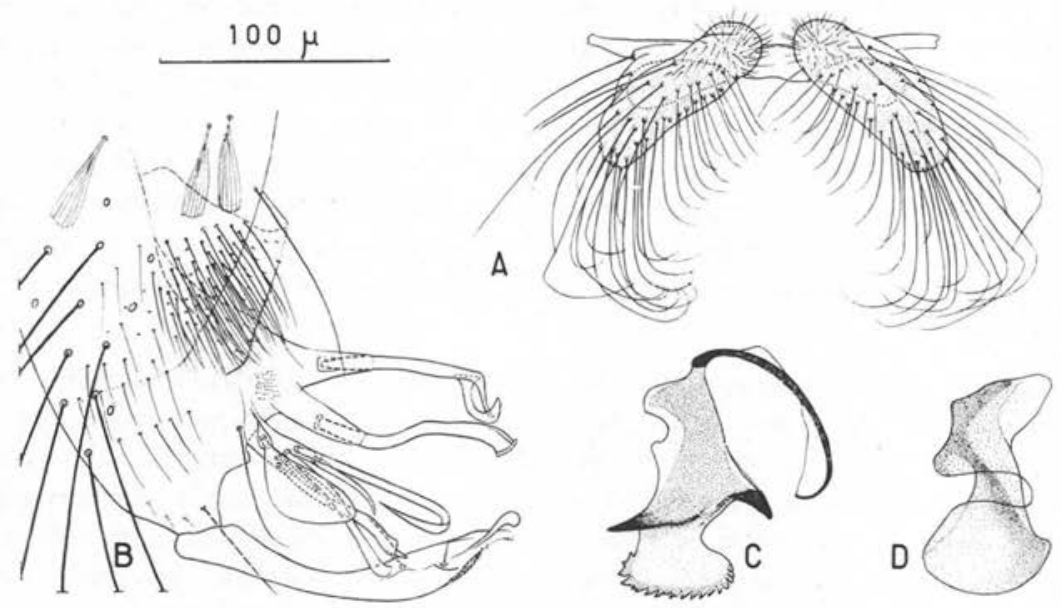

FIg. 1. - Pince génitale de Culex (Melanoconion) seneveti n. sp. A.: lobes du IXe tergite; B. : extrémité distale du coxite et style; $\mathrm{C}$ : plaque interne du mésonome ; D. : plaque externe du mésonome

Sur la division externe, les deux premiers filaments sont portés par une digitation bien individualisée; le premier long, relativement mince et terminé par un long crochet ; le deuxième de moitié plus court (crochet non compris), implanté beaucoup plus haut (distalement) et plus postérieurement (sur le plan de la figure) que le premier. Troisième filament prenant naissance directement sur la division, un peu plus bas (proximalement) que le premier, très long, large, à extrémité arrondie, légèrement recourbée. Sur tous les exemplaires examinés, ce filament croise le groupe des trois ou quatre filaments suivants, dont les implantations sont groupées à la même hauteur moyenne que la troisième. Dans la partie la plus interne de la base de la division externe prend naissance une formation sinueuse, semblable à une crête, qui se dirige suivant la diagonale correspondante en grossissant rapidement, jusqu'à former un tubercule subcylindrique. Ce tubercule porte à son extrémité une sorte de vaste cupule, dans le fond de laquelle s'implante une grande feuille striée (striation non représentée sur la figure), dont la forme varie suivant son orientation. Elle peut être grossièrement triangulaire comme chez le type, ou plus allongée, à bords presque parallèles. Le tubercule qui porte la feuille présente une petite encoche subapicale sur son bord interne. Un très long poil enfin prend naissance près de la base de la division externe, mais sur le coxite. 
Style sans caractère marquant. Sa moitié distale présente la forme dite en hure, qui se retrouve chez de nombreuses espèces; ici très allongée et peu grossie. Dans sa partie terminale, cette hure présente une série de striations transversales profondes, pouvant ressembler à des poils sous certaines incidences.

Plaque interne du mésonome d'une forme également observée chez plusieurs espèces pouvant avoir assez peu d'affinités entre elles. A la base du bord dorsal, un bec large, à extrémité arrondie, dirigé vers l'apex; à peine distal du précédent et lui faisant face, un autre bec, peu saillant et très arrondi ; plus distalement encore, un bras puissant, pointu, de même valeur que le bras du bord ventral. Ce dernier bien développé, légèrement coudé, situé au milieu du bord qui le porte (position légèrement variable suivant l'orientation de la plaque sur la préparation). Le bord apical enfin porte des denticulations larges et profondes, qui débordent sur le bord dorsal. Afin de permettre une éventuelle comparaison avec d'autres espèces voisines, et malgré sa faible valeur taxonomique, la plaque externe du mésonome a été représentée, en $D$, sur la figure.

Les lobes du $\mathrm{IX}^{\mathrm{e}}$ tergite sont d'une forme irrégulière et difficile à définir sur un schéma, car tous les plans y ont la même valeur. A l'examen microscopique au contraire, ils se détachent en premier plan et apparaissent très nettement (en grisé sur la figure) subcylindriques, plus de trois fois aussi longs que larges, distants l'un de l'autre à la base de la moitié de leur propre largeur, et de directions très fortement divergentes au-delà. La base des lobes représente une projection rétrograde, comme chez $C$. phlogistus. Elle porte de nombreux poils courts et fins, disposés sans ordre, non orientés, et approximativement limités par une ligne oblique correspondant au bord distal du tergite. Le quart distal des lobes porte au contraire un bouquet de poils longs, minces, rectilignes sauf à l'extrémité qui varie suivant leur position : les plus internes sont légèrement recourbés; dans la partie moyenne du bouquet ils forment un crochet; les plus externes présentent successivement une courbure serrée, une deuxième partie rectiligne, un crochet. Chez quelques-uns de ces derniers, l'une des deux parties rectilignes peut présenter une légère courbure inversée. Sur le bord interne, de nombreux poils longs, minces, dirigés vers la ligne médiane, rectilignes, sauf à leur extrémité qui est légèrement recourbée vers le tergite. Sur le bord externe et les expansions latérales du lobe, quelques longs poils raides, dont la direction est sensiblement parallèle à l'axe de ce lobe. Cette disposition, tout à fait caractéristique, se reconnaît immédiatement à l'examen, même à un très faible grossissement; elle persiste lorsque les lobes ont été aplatis et déformés par une excès de compression de la lamelle.

Culex seneveti prend place dans le groupe des Melanoconion dont la division externe du lobe du coxite porte une feuille et présente une disposition comparable des filaments, et dont le mésonome, denticulé sur le bord apical, varie peu d'une espèce à l'autre: C. phlogistus Dyar, 1920, C. plectoporpe Root, 1927, C. carcinophilus Dyar et Knab, 1906, C. inhibitator Dyar et Knab, 1906, C. wepsteri Komp et Rozeboom, 1951. 
Seul, $C$. phlogistus est souvent capturé en Guyane Française. D'après les échantillons examinés, il se sépare immédiatement de seneveti par une disposition différente des poils du $\mathrm{IX}^{\circ}$ tergite, par la base nue de ces lobes, ainsi que par l'insertion des deux premiers filaments de la division externe du lobe du coxite à un même niveau.

Chez $C$. plectoporpe, les lobes du $\mathrm{IX}^{e}$ tergite sont plus globuleux et la disposition des poils différente; les deux premiers filaments de la division externe du coxite sont insérés à un même niveau; le style présente enfin une expansion triangulaire subapicale surmontant la soie «oculaire » (d'après Root 1927 et Rozeboom et Komp 1950). Pour P. Fauran (1961 a), qui fait de plectoporpe un synonyme de phlogistus, ce dernier caractère résulterait d'un artéfact de préparation, la saillie étant visible sur les échantillons examinés sans compression, mais disparaissant, par suite d'une orientation différente, après mise en place de la lamelle. Que $C$. plectoporpe soit une espèce valide ou non est sans rapport direct avec le problème qui nous occupe ici.

Pour les deux espèces suivantes, nous nous référerons essentiellement à la publication de Rozeboom et Komp déjà citée.

C. carcinophilus présente sur le style une expansion de forme arrondie, caractéristique ; les deux premiers filaments de la division externe du coxite sont insérés à un même niveau. Les lobes du IX tergite ne sont ni décrits ni figurés par Rozeboom et Komp, mais, d'après le dessin de J. Lane (1953), ils sont petits, pas plus hauts que larges, dirigés vers la ligne médiane, ce qui est exactement l'inverse de ce qui s'observe chez seneveti.

Chez $C$. inhibitator, comme chez seneveti, l'insertion du deuxième filament de la division externe du coxite est distale de celle du premier; la feuille de cette division est portée sur un tubercule bien développé, près duquel s'insère un long poil ; la hure du style est allongée, peu grossie, et présente une forte striation subterminale. Les seules différences, minimes, que l'on puisse observer sur le dessin de Rozeboom et Komp sont : la plus grande longueur du deuxième filament, son insertion sur le même plan que le premier et peut-être un peu moins distale; la forme et la position du troisième filament, rectiligne, en lame pointue, restant placé entre le groupe de filaments qui le précède et celui qui le suit ; l'implantation de la feuille à l'extrémité du tubercule, et à peu près au même niveau que les filaments; l'insertion du poil accompagnant la feuille sur la division et non sur le coxite. La striation de l'extrémité du style enfin s'étend davantage en direction de la base. Il y a peu à dire sur le mésonome dont l'aspect, chez un même spécimen, peut présenter de légères différences d'un côté à l'autre. Notons seulement l'absence de becs dans la partie basale du bord dorsal; un bras moins fort dans la partie distale du même bord, la tendance des denticulations du bord apical à déborder sur le bord ventral plutôt que sur le bord dorsal. Les lobes du $\mathrm{IX}^{\circ}$ tergite sont subcylindriques, mais plus courts (deux fois et demie la largeur moyenne), très rapprochés l'un de l'autre; l'orientation générale de la pilosité, assez uniforme et raide, courte, se fait vers l'extérieur des lobes, disposition exactement inverse de celle de seneveti.

Chez $C$. wepsteri, qui porte également une touffe de longs poils dans la partie moyenne du bord ventral du coxite, tous les filaments de la division externe du lobe 
subapical sont semblables à ceux de seneveti. On peut cependant séparer ces espèces par les caractères suivants, notés chez la première (Komp et Rozeboom 1951) : bord interne du coxite concave; branche la plus interne de la division interne encore plus courte, presque sessile; deuxième filament de la division externe inséré sur le même plan que le premier; feuille ovoïde, prenant naissance directement sur la division, sans autre formation surajoutée (tige, tubercule); style présentant une fine pilosité dans sa partie distale; mésonome, dans les limites du seul échantillon examiné, de forme " rectangulaire », sans même une ébauche de bec dans la partie basale du bord dorsal ; lobes du $\mathrm{IX}^{\circ}$ tergite grossis dans leur partie médiane, elliptiques, nus à la base, couverts, partout ailleurs, de longs poils disposés d'une manière uniforme autour du lobe, en plumeau, et ne différant entre eux que par une minime question de longueur.

Forêt de Cabassou; pris au piège lumineux. A l'est de la route départementale, nuit du 4 au 5 septembre 1967: $3 \hat{\delta}\left(\mathrm{n}^{\circ}\right.$ 3248); nuit du 5 au 6 septembre: $1 \hat{o}$ $\left(\mathrm{n}^{\circ}\right.$ 3256) ; nuit du $1^{\text {er }}$ au 2 avril 1968: 4 ô $\left(\mathrm{n}^{\circ}\right.$ 3345. A l'ouest de la même route, nuit du 27 au 28 mars 1968: 3 ô ( $\left.\mathrm{n}^{\circ} 3328\right)$; soirée du 15 au 16 juin 1968: $1 \hat{o}$ $\left(n^{\circ} 3478\right)$. Le spécimen $n^{\circ} 3328-47$ a été choisi comme holotype.

\section{Culex (Melanoconion) abonnenci n. sp.}

MÂLE.

Antenne aussi longue que la trompe; flagelle, poils et verticilles d'un même brun très clair. Palpe plus long que la trompe, le milieu du quatrième article atteignant à peu près la base des labelles; noir. Clypeus brun noir ; trompe noire. Occiput comme chez l'espèce précédente. Mesonotum, scutellum, post-scutellum brun noirâtre, à écailles et poils noirs. Pleures brun noirâtre, sauf le pronotum postérieur qui est brun; progressivement plus claires dans leur partie distale. Aile, balancier, pattes, comme chez l'espèce précédente. Abdomen également semblable, avec de grosses taches basolatérales d'écailles blanches, bien visibles depuis le $\mathrm{II}^{\circ}$ segment.

Pince génitale (fig. 2). Coxite long et mince, piriforme, à sommet légèrement recourbé sur son axe. Division interne du lobe subapical longuement bifide, les deux branches fortement divergentes et de longueurs très inégales. Les filaments qu'elles portent subégaux et de même apparence, sinueux, le plus interne grossi dans sa partie moyenne, doublement recourbés dans leur partie distale et barrés en $T$ par une crête à leur extrémité.

Premier filament de la division externe long, mince, à crochet terminal très élargi. Deuxième filament beaucoup plus court et de moitié moins large que le précédent ; implanté au-dessus de celui-ci (sur le plan de la figure) et un peu plus distalement. Troisième filament proximal des deux précédents, fortement grossi dans sa moitié basale, progressivement plus mince dans sa moitié distale. Exactement au même niveau que le troisième filament, mais sur l'autre face de la division, prend naissance sur une base vigoureuse, une très grande feuille subtriangulaire, ondulée, striée à la base, qui attire le regard (striation non représentée sur la figure). Dans sa partie la plus externe et plus proximalement encore, cette division porte quatre 
filaments (trois disposés sur un rang, le quatrième au-dessous des précédents), dont la disposition générale est difficile à préciser et la représentation sur la figure approximative. La moitié basale de la division enfin est recouverte, du côté où se trouve la feuille (face cachée de la figure), par une expansion triangulaire du coxite, juxtaposée ou soudée, qui porte, sur sa face libre, un poil long et fort, de même valeur que celui qui se trouve à l'extrémité du coxite.
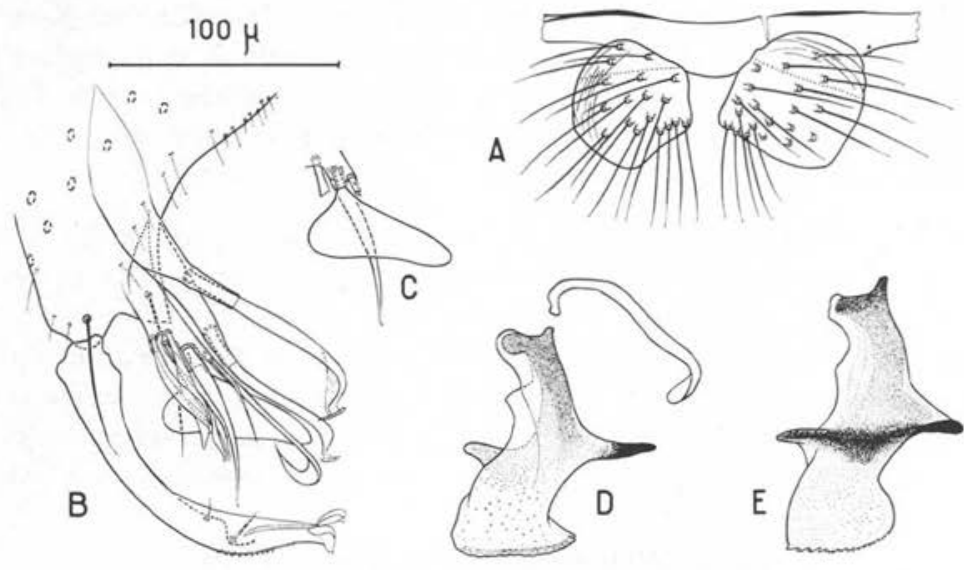

FIG. 2. - Pince génitale de Culex (Melanoconion) abonnenci n. sp. A. : lobes du IX IX $^{\circ}$ tergite ; B : extrémité distale du coxite et style ; C. : troisième filament et feuille de la division externe du deuxième coxite vus par l'autre face; D. : plaque interne du mésonome da l'holotype dont les denticulations ne sont pas visibles; E. : plaque interne du mésonome du paratype montrant les dents de scie du bord apical

Style en forme de hure, à peine grossi dans sa partie terminale et sans caractères particuliers, sinon le grand développement de la soie « oculaire ».

Plaque interne du mésonome de la même forme générale que pour l'espèce précédente. Le bras du bord dorsal y est en position submédiane et presque en face du bras ventral. Les denticulations du bord apical ne sont pas visibles sur le mésonome du type, représenté en $\mathrm{D}$ sur la figure. La plaque interne du mésonome du paratype a été représentée en $\mathrm{E}$, vue par son autre face; les dents de scie du bord apical, petites et peu profondes, $\mathrm{y}$ apparaissent très nettement.

Lobes du $\mathrm{IX}^{\circ}$ tergite petits, subglobuleux, nettement séparés l'un de l'autre. Poils peu nombreux, disposés principalement dans la partie interne et proximale des lobes, ménageant une large bande nue le long du bord externe; à base saillante; de longueur médiocre, raides et de directions plus ou moins radiées, de telle sorte que l'ensemble s'inscrit dans un rectangle transversal, le long du IX $^{\circ}$ tergite.

L'implantation du troisième filament et de la feuille de la division externe du coxite à un même niveau, entre le groupe des deux premiers filaments et celui des trois ou quatre derniers, se retrouve également chez C. comminutor Dyar, 1920, C. conspirator Dyar et Knab, 1906, C. distiguendus Dyar, 1928, C. implicatus Senevet 
et Abonnenc, 1939, C. intricatus Brèthes, 1916, C. mutator Dyar et Knab, 1906, C. sursumptor Dyar, 1924, C. thomasi Evans, 1924, dont les lobes du IX ${ }^{e}$ tergite sont aussi de petite taille, à pilosité rare, courte et raide. Mais, chez toutes ces espèces, la plaque interne du mésonome est d'une forme très différente.

Chez C. elevator Dyar et Knab, 1906 au contraire, qui présente une même disposition des filaments et de la feuille, la plaque interne du mésonome est denticulée sur son bord apical. Mais elle diffère de celle de $C$. abonnenci par l'absence de bras sur le bord ventral. Ce dernier caractère cité d'après les publications de Rozeboom et Komp (1950), Lane (1953), et d'après la description originale de deux espèces tombées en synonymie: $C$. bonneti Senevet, 1938 et $C$. vogelsangi Anduze, 1948. D'après ces mêmes auteurs, la feuille de $C$. elevator semble être de plus petite taille, plus allongée et moins apparente que chez abonnenci.

Forêt du Gallion. En bordure de la route nationale; soirée du 12 juin 1968 : $2 \hat{o}$ pris dans un piège lumineux $\left(\mathrm{n}^{\circ}\right.$ 3477). Spécimen $\mathrm{n}^{\circ} 3477-5$ choisi comme holotype.

Fauran $(1961 b)$ signale la présence de $C$. elevator en Guyane Française d'après un seul exemplaire collecté par E. Abonnenc et rapporté, sans une totale conviction semble-t-il à cette espèce. Il serait intéressant de revoir la préparation, ou les croquis laissés par Abonnenc, afin de préciser les rapports de cet exemplaire avec $C$. abonnenci.

\section{Culex (Melanoconion) caudatus $\mathrm{n} . \mathrm{sp}$.}

MÂLE.

Tête comme chez $C$. seneveti. Thorax entièrement d'un brun sombre uniforme ; écailles et poils du mesonotum et du scutellum noirs. Une touffe d'écailles blanches au niveau des soies sternopleurales inférieures et une autre au niveau des soies mésepimerales supérieures. Le reste, comme chez seneveti.

Pince génitale (fig. 3). Coxite en forme de cône allongé, recourbé sur son axe, particulièrement dans son tiers distal. Des rangées régulières de poils longs, forts et raides sur sa face postéro-externe.

Division interne du lobe subapical bien développée; ses deux branches longues, divergentes; la plus interne plus courte que l'externe. Premier filament rectiligne, doublement recourbé en crochet à son extrémité ; le deuxième présentant deux larges ondulations sur sa longueur et barré en $\mathrm{T}$ par une crête transversale à son extrémité.

Division externe (en grisé sur la figure) de grande taille, légèrement rétrécie dans sa partie médiane. Deux premiers filaments portés sur une même digitation de la division ; le premier long, rectiligne, recourbé en crochet à son extrémité ; le deuxième plus distal, moins long et moins fort que le précédent, à extrémité spatulée. Bases des filaments suivants proximales des deux précédentes, contiguës, alignées sur le bord distal de la division à un même niveau. Troisième filament à peu près de la même valeur que le premier, à extrémité légèrement massuée, pouvant simuler un crochet. Quatrième filament à peine moins gros que le précédent, terminé en pointe ; cinquième et sixième filaments plus grêles que le quatrième, à extrémité renflée. Comme chez 
l'espèce précédente, le coxite porte sur sa face postérieure une expansion triangulaire subapicale qui recouvre la moitié basale de la division externe. Cette expansion porte un très long poil terminal et un autre, plus court, dans la partie basale de sa face libre.

Style de la même forme que chez les espèces précédentes, mais relativement plus vigoureux ; la hure aussi longue que la moitié de l'article, à bords interne et externe rectilignes et parallèles.

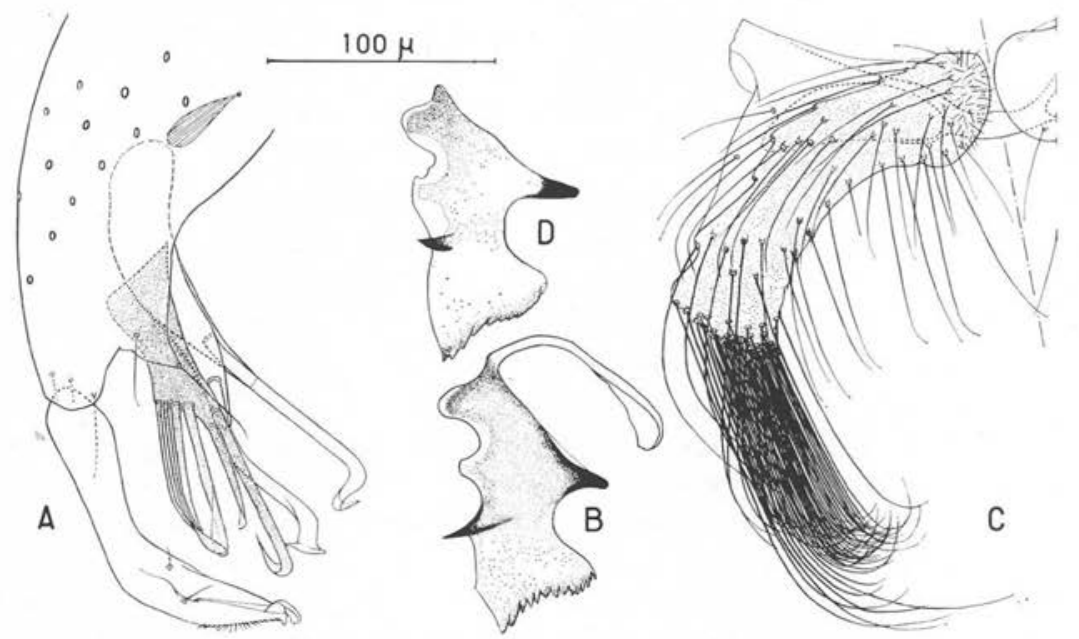

Fig. 3. - Pince génitale de Culex (Melanoconion) caudatus n. sp. A.: extrémité distale du coxite et style; B. : plaque interne du mésonome; C. : lobe gauche du IX tergite dont l'axe a été légèrement incliné sur la gauche; D. : plaque interne du mésonome du paratype

Plaque interne du mésonome (B) de la même forme que chez les espèces précédentes. Denticulations du bord apical larges, profondes, mousses; bras ventral extrêmement vigoureux ; bras dorsal plus faible, légèrement recourbé, finissant en pointe très aiguë. Entre ce bras et la base de la plaque, en position médiane, une saillie volumineuse et prononcée ; entre ce bras et les denticulations apicales, le bord dorsal n'est pas arrondi, mais forme, chez le type, un angle obtus très bien marqué. En $\mathrm{D}$, pour comparaison, la plaque interne du mésonome de l'autre exemplaire examiné.

La forme des lobes du $\mathrm{IX}^{\circ}$ tergite varie sensiblement suivant que la préparation est nue ou recouverte d'une lamelle, et suivant le degré de compression exercé par celle-ci. Dans la dernière éventualité, tous les plans tendent à se confondre, les lobes sont aplatis et déformés. Lorsqu'au contraire la préparation est nue, les lobes (en grisé sur la figure) se détachent nettement sur les parties annexes et apparaissent très longs (environ cinq fois aussi longs que la plus faible largeur), subcylindriques, courbés en arc de cercle, faiblement grossis à chaque extrémité, se touchant à la base et fortement divergents sur le reste. Base du lobe arrondie, couverte sur ses deux faces de 
poils courts, fins, disposés sans ordre, non orientés, dont les implantations sont à peine visibles. Extrémité distale du lobe rectiligne mais non transversale, légèrement oblique de l'extérieur vers la partie médiane de la pince et de la base vers l'apex. Elle porte des poils nombreux, implantés très obliquement sur son bord et dans le même sens que celui-ci, serrés, longs, vigoureux, raides, terminés en crochet et formant un pinceau dont la longueur reste inférieure à celle du lobe. Dans la partie la plus externe du pinceau, les poils sont plus longs et plus raides, à extrémité non crochue. L'ensemble ne présente pas une direction franchement antéro-postérieure, mais légèrement oblique vers la partie médiane de la pince. Sur son bord interne le lobe porte des poils très longs, grêles, rectilignes, régulièrement espacés, dont la direction générale reste sensiblement parallèle à celle du pinceau terminal. Sur le reste du lobe, des poils très longs, grêles, irrégulièrement répartis, parallèles à l'axe de ce lobe.

Cette espèce est très proche de $C$. serratimarge Root, 1927, qui s'en sépare par les caractères suivants, notés à la fois, sauf indication contraire, sur la publication originale de Root, celles de Rozeboom et Komp (1950), de J.-P. Duret (1953). Le deuxième filament de la division externe du lobe subapical du coxite est très grêle, presque filiforme ; il est inséré basalement par rapport au premier ; la distance séparant le deuxième filament du troisième est considérable et le bord de la division y apparaît libre et nu (Duret signale la présence d'un petit poil au milieu); le troisième filament est inséré sur une digitation propre du bord de la division; les trois derniers filaments (quatre pour Duret) sont insérés sur une autre digitation prenant naissance à la base de la division. La plaque interne du mésonome de $C$. serratimarge est partiellement représentée, et d'une interprétation difficile sur le dessin de Root ; elle est semblable à celle de caudatus sur le dessin de Rozeboom et Komp et très différente sur celui de Duret; elle ne peut donc nous être d'aucun secours. D'après les dessins de Root, Rozeboom et Komp, les lobes du IX $^{e}$ tergite sont massifs, à peine trois fois aussi longs que leur plus faible largeur, avec un rétrécissement en diabolo dans la partie médiane. Le bord distal est très légèrement oblique, dans le même sens que chez C. caudatus, pour Root, franchement transversal pour Rozeboom et Komp. Les poils du pinceau sont implantés perpendiculairement à la surface qui les porte, beaucoup plus longs que le lobe lui-même, et, pour le groupe interne, sans aucune tendance à former un crochet à leur extrémité. Les poils répartis sur le reste du lobe sont au contraire très courts, plus courts que la plus faible largeur de ce lobe.

C. caudatus est également très proche de $C$. isabelae Duret, 1968, qui s'en sépare par les caractères suivants : présence de deux poils, longs, minces, sinueux, très différents du reste de la pilosité, vers le milieu de la face externe du coxite; deuxième filament de la division externe du lobe subapical foliacé, terminé en pointe ; d'après la figure, base du troisième filament faisant saillie au-dessus du bord de la division, ce filament présentant une légère courbure sur toute sa longueur, grossi dans sa moitié basale, rétréci dans sa moitié distale, terminé en pointe mousse, non massué ; filaments quatre à six portés sur une digitation propre ; tubercule placé sur le côté externe de la division, que nous avons appelé expansion triangulaire du coxite, atteignant la base des quatre derniers filaments. Style plus grêle, le bord externe de la hure présentant une 
proéminence arrondie, qui donne à l'ensemble la forme d'une tête de cygne. Lobes du $\mathrm{IX}^{*}$ tergite bien séparés à la base, fortement rétrécis dans leur tiers médian, non subcylindriques; courte pilosité basale insérée sur des nodules triangulaires saillants ayant l'apparence de petites épines; pinceau terminal de poils proportionnellement plus court, onduleux dans sa partie distale et prenant une direction franchement transversale, vers son homologue du côté opposé; tous les autres poils nettement plus courts.

Forêt de Cabassou. A l'est de la route départementale; nuit du 21 au 22 août 1967: $1 \hat{\delta}\left(\mathrm{n}^{\circ}\right.$ 3242-15); nuit du 4 au 5 septembre 1967: 1 o $\left(\mathrm{n}^{\circ}\right.$ 3247-21) pris comme holotype.

G. Senevet et E. Abonnenc (1939) ont signalé la présence de $C$. serratimarge en Guyane Française d'après un exemplaire mâle, vraisemblablement obtenu d'élevage à partir d'une larve collectée dans le marais de Cabassou. Il est frappant de constater sur la figure que la forme de la division externe du lobe subapical du coxite ne concorde pas avec les descriptions de cette espèce déjà citées, mais se superpose au contraire très exactement à celle de $C$. caudatus. Les lobes du $\mathrm{IX}^{\bullet}$ tergite, le tergite lui-même vu par transparence, la répartition et la longueur des grands poils (non la disposition, peut-être modifiée par le montage) sont aussi très exactement les mêmes que chez l'espèce nouvelle, ce qui nous incite à penser que le Moustique de Senevet et Abonnenc doit revenir à $C$. caudatus plutôt qu'à $C$. serratimarge.

Dans le même ordre d'idées, il serait peut-être intéressant de revoir les récoltes ultérieures de $C$. serratimarge faites en Guyane Française par H. Floch et E. Abonnenc (1942, 1947), rappelées par Fauran (1961 b).

\section{Culex (Melanoconion) trigeminatus n. sp.}

MÀle.

Le corps de ce Moustique, pris dans un piège lumineux et en très mauvais état, a été jeté par inadvertance. La pince génitale seule sera décrite.

Pince génitale (fig. 4). Coxite conique, à bord externe légèrement convexe et bord interne rectiligne; non recourbé sur son axe ; deux fois aussi long que sa largeur à la base. Des rangées de poils longs et forts, ainsi qu'une touffe de poils longs et minces dans la partie médiane, sur la face postérieure (cachée, de la figure) ; pilosité courte et rare sur l'autre face (représentée dans sa totalité).

Le lobe subapical du coxite comprend trois groupes de deux éléments, sinon identiques du moins semblables ou de même nature, auxquels ce Moustique doit son nom.

Les deux premiers de ces groupes paraissent représenter la division interne en raison de leurs racines communes. Le premier groupe (plan supérieur de la figure) est formé de deux longues branches très inégales, portant chacune un filament vigoureux, cylindrique à la base, élargi et plus ou moins lancéolé au-delà, légèrement onduleux; le premier filament est terminé en pointe très acérée, le deuxième, deux fois plus large, en pointe mousse. Le deuxième groupe, au-dessous et légèrement distal 
du précédent, est aussi formé de deux branches inégales mais courtes, dont chacune porte un filament rectiligne, en forme de lame pointue, le deuxième filament étant deux fois plus large que le premier.

Le troisième groupe, plus apical que les deux autres, représente la division externe. Il comprend une longue colonne cylindrique dont l'extrémité basale, en bec de flûte, épouse la surface arrondie du coxite, tandis que l'autre extrémité porte sur une face une très grande et large feuille striée, en position subapicale, et quatre filaments terminaux sur l'autre face. Une autre colonne beaucoup plus courte, ou tubercule, à base très élargie à la manière d'un écusson, coalescente avec la première, porte à son extrémité distale une longue feuille lancéolée, striée, à très long pétiole (striation omise pour les deux feuilles sur la figure).

Style droit, rectiligne dans ses deux tiers basaux, très légèrement grossi et coudé vers la base de la pince dans son tiers distal.

Plaque interne du mésonome se rapprochant de celles de $C$. dunni, C. zeteki, mais avec un développement considérable du bord ventral, qui lui donne une forme triangulaire, la largeur de la plaque (sens dorso-ventral) restant toutefois inférieure à sa hauteur (sens baso-apical); ce bord entièrement dépourvu de spicules. Un bec volumineux, arrondi, à la base du bord dorsal ; une légère proéminence mousse vers le milieu du même bord. Apex pointu, en bec, avec divers épaississements linéaires longitudinaux qui en soulignent les contours et lui confèrent un aspect de robustesse.

Afin de permettre une éventuelle comparaison avec des espèces voisines, la plaque externe et la plaque basale du mésonome ont été représentées, respectivement en $\mathrm{D}$ et $\mathrm{E}$ sur la figure. Ainsi, l'extrémité effilée de la plaque basale est rectiligne chez C. trigeminatus, tandis qu'elle présente une double ondulation de grande amplitude chez $C$. dunni; elle est beaucoup plus large et coudée comme un pied sur la jambe chez $C$. zeteki.

Lobes du $\mathrm{IX}^{e}$ tergite en ellipsoïdes, largement séparés à la base et de directions fortement divergentes. Poils approximativement groupés sur le troisième quart du lobe, sur toute sa largeur (sur le lobe droit, où ils sont moins abondants, ils forment une bande transversale); de longueur moyenne, raides, presque tous avec une ou deux ramifications dendritiques à leur extrémité, visibles seulement à un très fort grossissement (ramifications semblables observées chez $C$. dunni et chez $C$. zeteki).

C. trigeminatus présente certaines affinités avec C. dunni Dyar, 1918 et C. zeteki Dyar, 1918. Mais, chez ces deux espèces, la division interne du lobe subapical du coxite compte seulement trois filaments, en baguettes; la deuxième feuille est portée directement sur le coxite, à une grande distance de la première ; l'extrémité du style est effilée, la plaque interne du mésonome spiculée chez $C$. dunni; l'extrémité du style doublement recourbée, la plaque interne du mésonome d'une forme différente chez $C$. zeteki. (D'après l'examen d'exemplaires capturés en Guyane Française ainsi que d'après Rozeboom et Komp 1950).

Les affinités de $C$. trigeminatus avec C. caribeanus Galindo et Blanton, 1954 sont au contraire beaucoup plus étroites. Ce dernier est caractérisé par: division interne du coxite formée de trois branches groupées au même niveau, portant chacune 
un filament, le deuxième de ces filaments avec une crête longitudinale saillante sur presque toute sa longueur. Première feuille de la division externe non subapicale mais insérée vers le milieu de la colonne; deuxième feuille implantée directement sur le coxite, moins nettement lancéolée, à pétiole moins long. Bord ventral de la plaque interne du mésonome encore plus développé, la largeur de celle-ci dépassant sa hauteur ; bec apical plus long, plus mince ; ces divergences ne paraîssant pas être sous la dépen-
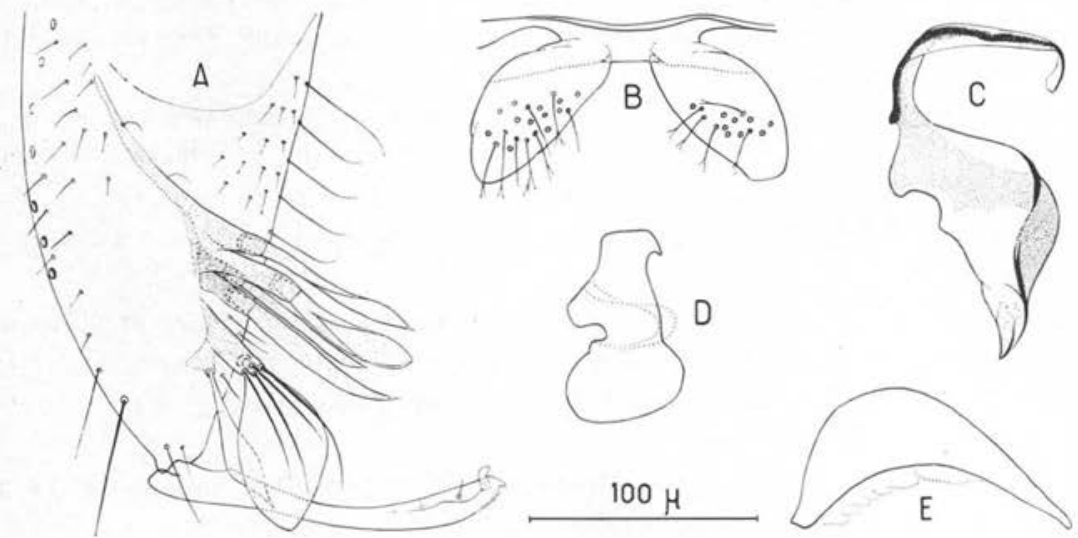

FIG. 4. - Pince génitale de Culex (Melanoconion) trigeminatus n. sp. A. : extrémité distale du coxite et style ; B.: lobes du IX tergite ; C.: plaque interne du mésonome ; D. : plaque externe du mésonome; E. : plaque basale du mésonome

dance d'une orientation différente des plaques sur les préparations. Lobes du IX ${ }^{\circ}$ tergite plus régulièrement rétrécis de la base au sommet, terminés en pointe mousse ; poils occupant presque toute la hauteur des lobes, sur une étroite bande, le long du bord interne.

Forêt du Gallion. Nuit du 19 au 20 avril 1968 ; pris au piège lumineux : $1 \hat{\jmath}$ ( $\mathrm{n}^{\circ}$ 3381-1) constituant l'holotype.

\section{Bibliographie}

Anduze (P. J.), 1948. - Fauna Culicina de Venezuela. Descripcion de cinco nuevas espécies. Bol. ent. Venezol., 7, 60-66.

Duret (J. P.), 1953. - Las espécies argentinas de Culex (Melanoconion) (Diptera, Culicidae). Rev. Soc. ent. Arg., 16, 99-121.

-, 1967. - Cinco espécies nuevas de Culex (Melanoconion) (Diptera, Culicidae). Rev. Soc. ent. Arg., 30, 69-81.

Fauran (P.), 1961 a. - Notes sur les Moustiques de la Guyane. Arch. Inst. Pasteur Guyane Fr., Publ. 464, 15 p. 
-, 1961 b. - Catalogue annoté des Culicidés signalśs en Guyane Française. Arch. Inst. Pasteur Guyane Fr., Publ. 465, 60 p.

Floch (H.) et ABonnenc (E.), 1942. - Catalogue et distribution géographique des Moustiques de la Guyane Française actuellement connus. Arch. Inst. Pasteur Guyane Fr., Publ. 43, $10 \mathrm{p}$.

—, - 1947. - Distribution des Moustiques du genre Culex en Guyane Française. Arch. Inst. Pasteur Guyane Fr., Publ. 146, 8 p.

Galindo (P.) et Blanton (F. S.), 1954. - Nine new species of neotropical Culex, eight from Panama and one from Honduras (Diptera, Culicidae). Ann. ent. Soc. Amer., 47, 231-247.

Komp (W. H. W) et Rozeboom (L. E.), 1951. - Descriptions of eight new species of Culex subgenus Melanoconion (Diptera, Culicidae). Proc. ent. Soc. Wash., 53, 121-137.

LANe (J.), 1953. - Neotropical Culicidae, T. 1, 548 p., Univ. de S. Paulo édit.

Root (F. M.), 1927. - Studies on Brazilian Mosquitoes. III. The genus Culex. Amer. Jl Hyg., 7, 574-598.

Rozeboom (L. E.) et Komp (W. H.W.), 1950. - A Review of the species of Culex of the subgenus Melanoconion (Diptera, Culicidae). Ann. ent. Soc. Amer., 43, 75-114.

Senevet (G.), 1938. - Les Moustiques de la Guadeloupe (Mission 1936). Arch. Inst. Pasteur Algérie, 16, 176-190.

-, et Abonnenc (E.), 1939. - Les Moustiques de la Guyane Française. II. Le genre Culex. Arch. Inst. Pasteur Algérie, 17, 62-134. 